\title{
ESPÉCIES DE THELAZIINAE (SKRJABIN) BAYLIS \& DAUBNEY (NEMATODA, THELAZIOIDEA) REFERIDAS PARA O BRASIL
}

\author{
Henrique de Oliveira Rodrigues ${ }^{1}$
}

\begin{abstract}
Species of Thelazinae Skrjabin (Nematoda, Thelazioidea) REPORTED FROM BRAZIL. Twenty three species of Thelaziinae Skrjabin, 1915 were referred in Brazil. Hempelia hempeli Vaz, 1936, Thelazia anolabiata (Molin, 1860), T. aquilina Baylis, 1934, T. campanulata (Molin, 1858), T. iheringi Travassos, 1918 and T. travassosfreitasi Cristofaro \& Rodrigues, 1979. That included in the Oswaldo Cruz Helminthological Collection were reviewed. Dryocopus lineatus lineatus (Linnaeus), Pipile cumanensis nattereri Reich and Ramphastus dicolorus Linnaeus are new host records for Thelazia anolabiata (Molin, 1860) and Rostrhamus sociabilis (Vieill.) is a new host record for Thelazia campanulata (Molin, 1858).

KEY WORDS. Nematoda, Thelaziinae, Hempelia, Thelazia, Ceratospira, Brazil
\end{abstract}

Em continuação aos estudos sobre Thelaziidae Skrjabin, 1915, foram reunidas neste trabalho as espécies de Thelaziinae (Skrjabin, 1915) Baylis \& Daubney, 1926 que foram registradas no Brasil.

Esta subfamília de nematóides parasitos de olhos de mamíferos e aves tem mais de meia centena de espécies descritas em todo o mundo, sendo algumas de interesse médico, já que foram encontradas parasitando o homem, como é o caso de Thelazia callipedae Railliet \& Henry, 1910 e T. californiensis Price, 1930.

Foram reunidas referências sobre 23 espécies citadas para o Brasil, sendo que aquelas que apresentavam material depositado na Coleção Helmintológica do Instituto Oswaldo Cruz (CHIOC) foram reestudadas.

As espécies indeterminadas aqui relacionadas que foram citadas por outros autores e que têm material depositado na $\mathrm{CHIOC}$, não puderam ser identificadas por se tratarem de fềmeas ou por não estarem em boas condições.

\section{MATERIAL E MÉTODOS}

$\mathrm{O}$ material existente na $\mathrm{CHIOC}$ foi revisto e muitas das amostras ainda não determinadas e as que estão em meio líquido, foram identificadas após serem submetidas aos processos usuais de diafanização pelo ácido acético glacial, fenol e creosoto de faia, sendo, após os estudos, recolocadas no líquido conservador (líquido de Railliet \& Henry). O material conservado em bálsamo foi também reestudado.

1) Departamento de Helmintologia, Instituto Oswaldo Cruz. Caixa Postal 926, 21041-210 Rio de Janeiro, Rio de Janeiro, Brasil. Bolsista do CNPq. 


\section{RESULTADOS E DISCUSSÃO}

Thelaziinae (Skrjabin, 1915) Baylis \& Daubney, 1926, encerra os gêneros: Hempelia Vaz, 1936, Ceratospira Schneider, 1866 e Thelazia Bosc, 1819.

No Brasil foram referidas as seguintes espécies:

Hempelia cameroni (Strachan, 1957) Rodrigues, 1980

Sinonímia: Oxyspirura cameroni Strachan, 1957.

Hospedeiro e proveniência: Tityra cayana Gambel, 1849 (=Piaya cayana macroura Gambel, 1849), Brasil.

Localização: olho.

Considerações: tipos depositados no Museum of Comparative Zoology, Harward University, USA.

\section{Hempelia hempeli Vaz, 1936}

Hospedeiros e proveniência: Nothura maculosa (Temm., 1815) e Rhynchotus rufescens (Temm., 1815), Itararé, São Paulo, Brasil.

Localização: olho e sob a membrana nictitante.

Considerações: tipos depositados na Coleção Helmintológica do Instituto Biológico, São PAulo, com os números 232, 239, 256 e 258. O parátipo número 32682 da CHIOC foi aqui revisto, tratando-se de um exemplar fêmea, em bom estado de conservação.

\section{Ceratospira thriponaxis Wehr, 1930}

Hospedeiros e proveniência: Ramphastus culminatus Gould, 1833, Brasil central.

Localização: olho.

Considerações: STRACHAN (1957) encontrou a espécie em Ramphastus culminatus Gould, 1833 proveniente do Brasil central sendo o material estudado, depositado no Museum of Comparative Zoology, Harward University, USA.

\section{Thelazia anolabiata (Molin, 1860) Railliet \& Henry, 1910}

Sinonímia: Spiroptera anolabiata Molin, 1860; Spiroptera cracis alectoris Molin, 1860; Filaria anolabiata Stossich, 1897×; Oxyspirura anolabiata Ramson, 1904; Thelazia anolabiata Railliet \& Henry, 1910; Thelazia (Thelazia) lutzi Travassos, 1918; Thelazia digitata Travassos, 1918; Thelazia anadorhynchi Strachan, 1957; Thelaziella anolabiata Skrjabin, Sobolev \& Ivaschkin, 1967; Thelaziella digitata Skrjabin, Sobolev \& Ivaschkin, 1967.

Hospedeiros e proveniência: Anadorhynchus hyacinthinus (Lathan, 1790), Brasil central; Crax fasciolata Spix, 1825, Barranco Alto, Rio Aquidauana, Mato Grosso do Sul; Dryocopus lineatus lineatus (Linnaeus, 1766) [=Ceophloeus lineatus lineatus (Linnaeus, 1766)], Bodoquena, Mato Grosso do Sul; Ortalis canicolis pantanalensis Cher. \& Reich., 1921, Salobra, Mato Grosso do Sul; Penelope superciliaris superciliaris Temminck, 1815, Brasil central; Penelope sp., Jacutinga, São Paulo; Pipile cumanensis nattereri Reich, 1862 [=Pipile grayi (Pelz., 1870)], Salobra e Barranco Alto, Mato Grosso do Sul; Pteroglossus aracari (Lin- 
naeus, 1758), Estrada Belém-Brasília, Pará; Ramphastus dicolorus Linnaeus, 1766, Araçatuba, São Paulo; Ramphastus tucanus Linnaeus, 1758, Estrada Manaus-Itacoatiara, Amazonas; Ramphastus sp., Brasil.

Localização: olho e sob a membrana nictitante.

Considerações: Molin (1860) estudando material de olhos de Crax fasciolata Spix, 1825, coletado por Natterer no Brasil, descreveu a espécie Spiroptera anolabiata, sendo os tipos depositados no Museu de Viena, Áustria.

Vários autores (DRASCHE 1883; ANDERSON \& DIAZ UNGRIA 1959; RODRIGUES \& RODRIGUES 1970; RoDRIGUES 1992) reestudaram essa espécie.

STRACHAN (1957) descreveu T. anadorhynchi, sendo o material tipo estudado, coletado em Anadorhynchus hyacinthinus (Lathan, 1790) e Penelope superciliaris superciliaris Temm., 1815, proveniente do Brasil central, o qual está depositado no Museum of Comparative Zoology, Harward University, USA.

ANDERSON \& DIAZ UNGRIA (1959), consideraram T. anadorhynchi idêntica a $T$. digitata e $T$. anolabiata idêntica a $T$. lutzi. RoDRIGUES (1992) considerou $T$. digitata, $T$. lutzi e $T$. anadorhynchi idênticas a $T$. anolabiata.

O material aqui estudado, identificado como T. anolabiata, está depositado na CHIOC com os números 184 (parasito de Ramphastus sp.), 6190 (parasito de Ramphastus dicolorus), 11592, 12450 e 14193 (parasito de Pipile cumanensis nattereri), 13300 (parasito de Dryocopus lineatus lineatus), 14556, 15535 e 15598 (parasitos de Crax fasciolata) em líquido de Railliet \& Henry e 30464 a-i (parasito de Pteroglossus aracari), 30499 a-b (parasito de Crax fasciolata), 30532 a-f (parasito de Penelope sp.), 32031 (parasito de Ramphastus tucanus), 32735 a-d, 32736 a-b, 32738 a-i, 32739 a-j, 32740 a-b e 32741 a-c (parasitos de Ortalis canicolis pantanalensis) em bálsamo.

Dryocopus lineatus lineatus, Pipile cumanensis nattereri e Ramphastus dicolorus são pela primeira vez referidos como hospedeiros da espécie.

\section{Thelazia aquilina Baylis, 1934}

Sinonímia: Thelazia chui Hsü, 1835; Thelazia spizaeti Strachan, 1957.

Hospedeiros e proveniência: Spizaetus ornatus (Daudin, 1801), Brasil central; Accipiter bicolor pileatus (Temm., 1823), Barranco Alto e Salobra, Mato Grosso do Sul Milvago chimachima chimachima (Vieil., 1816), Barranco Alto e Salobra, Mato Grosso do Sul; Polyborus plancus plancus (Miller, 1777), Barranco Alto, Mato Grosso do Sul; Buteogallus urubitinga urubitinga (Gm., 1788), Salobra, Mato Grosso do Sul.

Localização: olho.

Considerações: STRACHAN (1957) propôs uma nova espécie, Thelazia spizaeti, parasita de Spizaetus ornatus, proveniente do Brasil central. ANDERSON \& DIAZ UNGRIA (1959) revendo os tipos, consideraram a espécie idêntica a $T$. aquilina. PINTO et al. (1994), referiram a espécie em Accipiter bicolor pileatus, Buteogallus urubitinga urubitinga, Milvago chimachima chimachima e Polyborus plancus plancus, provenientes de Barranco Alto e Salobra, Mato Grosso do Sul.

O material estudado por Strachan e revisto por Anderson \& Diaz Ungria, 
está depositado no Museum of Comparative Zoology, Harward University, USA. $\mathrm{O}$ material estudado por Pinto et al. está depositado na CHIOC com os números 15078 e 15512 (em meio líquido) e 33073 e 33077 (em bálsamo).

\section{Thelazia buteonis Herde, 1942}

Hospedeiro e proveniência: Pipile cumanensis cumanensis (Jacquim, 1784), Brasil central.

Localização: olho.

Considerações: STRACHAN (1957) encontrou a espécie em material proveniente do Brasil Central o qual foi depositado no Museum of Comparative Zoology, Harward University, USA.

\section{Thelazia campanulata (Molin, 1858) Railliet \& Henry, 1910}

Sinonímia: Filaria falconis magnirostris Molin, 1858; Filaria campanulata Molin, 1858.

Hospedeiros e proveniência: Crypturellus strigulosus strigulosus Temminck, 1815, Brasil Central; falconídeo, São Paulo; Rupornis magnirostris superciliaris (Vieill., 1816), Salobra, Mato Grosso do Sul; Rostrhamus sociabilis (Vieill., 1817), Angra dos Reis, Rio de Janeiro.

Localização: olho.

Considerações: esta espécie foi descrita por MoLIN (1858), de material de Falco magnirostris $\mathrm{Gm}, 1789$ [=Rupornis magnirostris (Gm., 1789)] do Brasil, cujos tipos foram depositados no Museu de Viena, Áustria. Travassos (1918), redescreveu-a de uma espécie não determinada de Falconidae, proveniente de São Paulo, material este de número 229 da CHIOC que foi aqui revisto. Foram estudadas, também, amostras de Rupornis magnirostris superciliaris de números 11618 , 11678 e 11693 da CHIOC, proveniente de Salobra, Mato Grosso do Sul e de Rostrhamus sociabilis de número 1947, proveniente de Angra dos Reis, Rio de Janeiro que representa um novo hospedeiro e ampliação da distribuição geográfica da espécie.

STRACHAN (1957) identificou esta espécie em material proveniente do Brasil Central, parasita de Crypturelus strigulosus strigulosus que foi depositado no Museum of Comparative Zoology, Haward University, USA.

\section{Thelazia cholodkowskii Skrjabin, 1922}

Hospedeiro e proveniência: Otus choliba decussatus (Licht., 1823), Brasil Central.

Localização: olho.

Considerações: STRACHAN (1957), referiu a espécie em material proveniente do Brasil Central, o qual foi depositado no Museum of Comparative Zoology, Harward University, USA.

\section{Thelazia chungkingensis Hsü, 1933}

Hospedeiro e proveniência: Mitu mitu Linnaeus, 1766, Brasil Central.

Localização: olho

Considerações: STRACHAN (1957), identificou esta espécie estudando mate- 
rial proveniente do Brasil Central, o qual foi depositado no Museum of Comparative Zoology, Harward University, USA.

\section{Thelazia dacelonis (Breinl, 1913) Travassos, 1918}

Sinonímia: Filaria dacelonis Breinl, 1913

Hospedeiros e proveniência: Trogon melanurus melanurus Swainson, 1838 e Tinamus tao tao Temminck, 1815, Brasil Central.

Localização: saco conjuntival.

Considerações: STRACHAN (1957) referiu a espécie em material proveniente do Brasil Central, sendo as amostras estudadas depositadas no Museum of Comparative Zoology, Harward University, USA.

\section{Thelazia iheringi Travassos, 1918}

Hospedeiros e proveniência: Dasyprocta sp., Pirapora, Minas Gerais; Dasyprocta azarae, (Temminck, 1825), Salobra, Mato Grosso do Sul.

Localização: olho.

Considerações: Esta espécie foi descrita por TRAVASSOS (1918), de olho de Dasyprocta sp., proveniente de Pirapora, Minas Gerais, sendo os tipos depositados na Coleção Helmintológica do Museu Paulista, com o número 271 e os parátipos na CHIOC com os números 6321 e 1173 a-b. CRISTOFARO \& RODRIGUES (1979), referiram a espécie em Dasyprocta azarae proveniente de Salobra, Mato Grosso do Sul, material depositado na CHIOC com os números 31497, 31498 e 31499.

\section{Thelazia lacrymalis (Gurlt, 1831) Railliet \& Henry, 1910} 1844.

Sinonímia: Filaria lacrymalis Gurlt, 1831; Filaria palpebralis Wilson,

Hospedeiro e proveniência: Equus caballus Linnaeus, 1758, Brasil.

Localização: olho e condutos lacrimais.

Considerações: esta espécie foi referida no Brasil por TRAVASSOS (1918) e FreITAS \& COSTA (1970).

\section{Thelazia papillosa (Molin, 1860) Railliet \& Henry, 1910}

Sinonímia: Spiroptera papillosa Molin, 1860; Oxyspirura papillosa Ransom, 1904.

Hospedeiros e proveniência: Falco destructor Linnaeus, 1758 [=Thrasactus harpyia (Linnaeus, 1758)], Falco gracilis Temminck, 1821 [=Geranospiza caerulescens (Vieill., 1817)], Brasil.

Localização: olhos e sob a membrana nictitante.

Considerações: a espécie foi descrita por MoLIN (1860) estudando material coletado por Natterer no Brasil. Tipos depositados no Museu de Viena, Áustria.

\section{Thelazia pittae Johnston \& Mawson, 1941}

Hospedeiro e proveniência: Spizastur melanoleucus (Vieill., 1816), Brasil Central.

Localização: olho.

Considerações: STRACHAN (1957) referiu a espécie em material proveniente 
do Brasil Central, sendo as amostras estudadas depositadas no Museum of Comparative Zoology, Haward University, USA.

Thelazia sicki Strachan, 1957

Hospedeiro e proveniência: Otus sp., Brasil Central.

Localização: olho.

Considerações: tipos depositados no Museum of Comparative Zoology, Harward University, USA.

Thelazia tonkinensis Hsü, 1935

Hospedeiro e proveniência: Cephalopterus ornatus (Daudin, 1801), Brasil Central).

Localização: olho

Considerações: STRACHAN (1957) referiu a espécie em amostras provenientes do Brasil Central, as quais foram depositadas no Museum of Comparative Zoology, Haward University, USA.

\section{Thelazia travassosfreitasi Cristofaro \& Rodrigues, 1979}

Hospedeiro e proveniência: Dasyprocta azarae, Salobra, Mato Grosso do Sul.

Localização: olho.

Considerações: tipos depositados na CHIOC com os números 31500 a (holótipo), 31500 b-c e 31501 (parátipos).

\section{Thelazia sp. de Travassos et al. (1939)}

Hospedeiros e proveniência: Crax blumenbachii Spix, 1825, Ramphastus toco toco Muell., 1776, Trogonurus variegatus behni (Gould, 1875), Ortalis sp., Salobra, Mato Grosso do Sul.

Localização: olho.

Considerações: material estudado depositado na CHIOC com os números 11334, 11340, 11374, 11688 e 11689.

\section{Thelazia sp. de Travassos (1941)}

Hospedeiro e proveniência: Odontriorchis pileatus (Temminck, 1823), Salobra, Mato Grosso do Sul.

Localização: olho.

Considerações: material estudado depositado na CHIOC com o número 13013.

\section{Thelazia sp. de TRAVASSOS \& FREITAS (1941)}

Hospedeiros e proveniência: Rupornis magnirostris superciliaris, Milvago chimachima chimachima, Ortalis canicolis pantanalensis, Salobra, Mato Grosso do Sul.

Localização: olho.

Considerações: material estudado depositado na CHIOC com os números 11618,11678 e 11693 . 


\section{Thelazia sp. de TRAVAssos \& FREITAS (1942)}

Hospedeiro e proveniência: Buteo nitidus nitidus (Lath. 1790), Bodoquena, Mato Grosso do Sul.

Localização: olho. 13168 .

Considerações: material estudado depositado na $\mathrm{CHIOC}$ com o número

\section{Thelazia sp. de STRACHAN (1957)}

Hospedeiro e proveniência: Daptrius americanus (Boddaert, 1783), Brasil. Localização: olho.

Considerações: material estudado depositado no Museum of Comparative Zoology, Haward University, USA.

Thelazia sp. de PINTO et al. (1993)

Hospedeiro e proveniência: Amazona aestiva (Linnaeus, 1758), Barranco Alto, Mato Grosso do Sul.

Localização: olho.

Considerações: material estudado depositado na CHIOC com o número 32870 .

\section{REFERÊNCIAS BIBLIOGRÁFICAS}

ANDERSON, R.C. \& C. DiAz Ungria. 1959. Revision preliminar de las espécies de Thelazia Bosc (Spiruroidea, Thelaziidae) parasitas de aves. Mem. Soc. Ci. Nat. La Salle 19 (52): 37-75.

Cristofaro, R. \& H.O. Rodrigues. 1979. Sobre dois nematódeos do gênero Thelazia Bosc, 1819 parasitos de Dasyprocta azarae (Reng.) (Nematoda, Thelazioidea). Atas Soc. Biol. Rio de Janeiro 20: 7-9.

DRASCHE, R. 1883. Revision der in der Nematoden. Sammlung des k.k. Zool. Hofcabinetes befindlichen Original Exemplare Diesing's und Molin's. Verhandl. k.k. zool-bot. Ges. Wien 32: 117-138.

FreitAs, M.G. \& H.M.A. CostA. 1970 Lista dos helmintos parasitos dos animais domésticos do Brasil. Arq. Esc. Vet. 22: 33-94.

Molin, R. 1858. Versuch einer Monographie der Filarien. Sitzungsber. D. K. Akad. Wissench., Wien. Math.-Naturw. C.I. 28: 365-461. 1860. Uma monografia del genere Spiroptera. Sitzung Akad. Wissench., Wien. Math.-Naturw. 28: 911-1005.

PINTO, R.M.; J.J. VICENTE \& D. NoRONHA. 1993. Nematode parasites of Brazilian psittacid birds, with emphasis on the genus Pelecitus Railliet \& Henry, 1910. Mem. Inst. Oswaldo Cruz 88 (2): 279-284.

1994. Nematode parasites of Brazilian accipitrid and falconid birds (Falconiformes). Mem. Inst. Oswaldo Cruz 89 (3): 359-362.

Rodrigues, H.O. 1992. On Thelazia anolabiata (Molin, 1860) Railliet \& Henry, 1910 (Nematoda, Thelazioidea). A new host record and systematic considerations. Mem. Inst. Oswaldo Cruz 87 (Supl. 1): 217-222. 
Rodrigues, H.O. \& S.S. Rodrigues. 1970. Redescrição de Thelazia anolabiata (Molin, 1860) (Nematoda, Spiruroidea). Atas Soc. Biol. Rio de Janeiro 12 (Supl.): 29-31.

STRACHAN, A.A. 1957. Eye worms of the family Thelaziidae from Brazilian birds. Can. J. Zool. 35: 179-187.

Travassos, L. 1918. Contribuição para o conhecimento da fauna helmintológica brasileira. VII. Espécies brasileiras do gênero Thelazia Bosc, 1819. Rev. Museu Paulista 10: 215-230.

1941. Relatório da quarta excursão do Instituto Oswaldo Cruz à zona da Estrada de Ferro Noroeste do Brasil, realizada em agosto de 1940. Mem. Inst. Oswaldo Cruz 35 (4): 697-722.

Travassos, L. \& J.F.T. Freitas. 1941. Relatório da excursão científica realizada na zona da Estrada de Ferro Noroeste do Brasil, realizada em julho de 1939. Mem. Inst. Oswaldo Cruz 33 (3): 525-556. 1942. Relatório da sexta excursão do Instituto Oswaldo Cruz, realizada à zona da Estrada de Ferro Noroeste do Brasil, em novembro de 1941. Mem. Inst. Oswaldo Cruz 37 (3): 259-286.

Travassos, L.; H. LENT \& J.F.T. FreitAs. 1939. Relatório da excursão científica do Instituto Oswaldo Cruz, realizada na zona da Estrada de Ferro Noroeste do Brasil em outubro de 1938. II. Pesquisas helmintológicas. Bol. Biol. 4 (2): 221-249.

Recebido em 27.III.1995; aceito em 20.VIII.1996 